Hydrology and Earth System Sciences, 3(1), 85-93 (1999) (C) EGS

\title{
Functional similarity in landscape scale SVAT modelling
}

\author{
Keith J. Beven and Stewart W. Franks*†
}

Centre for Research on Environmental Systems and Statistics, Institute of Environmental and Natural Science, Lancaster University, Lancashire, LAl 4YQ U.K.

* Now at: Department of Civil, Surveying and Environmental Engineering, University of Newcastle, Callaghan 2308, NSW, Australia.

$\uparrow$ Corresponding author

\section{Abstract}

In this study, it is shown that the complexity of Soil Vegetation Atmosphere Transfer (SVAT) models leads to an equifinality of functional behaviour-many parameterizations from many areas of the parameter space lead to very similar responses. Individual parameters derived by calibration (i.e. model inversion) against limited measurements are, therefore, highly uncertain. Due to the non-linear internal behaviour of SVAT models, aggregation of uncertainly known parameter fields to parameterize landscape scale variability in surface fluxes will yield highly uncertain predictions. A disaggregation approach suggested by Beven (1995) requires that the land surface be represented by a linear sum of a number of representative parameterizations or functional types. This study explores the nature of the parameter space in terms of a simple definition of functional behaviour. Parameter interactions producing similar predicted behaviours are investigated through application of Principal Component Analyses. These reveal the lack of a dominant global interaction indicating the presence of highly complex parameter interactions throughout the feasible parameter space.

\section{Introduction}

There are many problems in estimating fluxes between the land surface and the atmosphere at the landscape scale (taken here to indicate the scale of a mesoscale or global circulation model grid element). Much of the discussion and theoretical development in the past has centred on the possibility of using 'effective' parameter values in a landscape scale SVAT (soil vegetation atmosphere transfer) model; values that are effective in the sense of taking account of all the local scale heterogeneities of soil and vegetation type, topographic position, surface roughness, water stress, and meteorological variables that influence the landscape scale integrated fluxes. Until recently, all of the SVAT models that have been linked to atmospheric circulation models have been of this type in which a onedimensional (vertical) SVAT representation has been used, with effective parameter values, to represent the surface of a whole grid element. Some of these models show considerable complexity in vertical structure (e.g. SiB, Sellers $e t$ al., 1986; and BATS, Dickinson and Kennedy, 1991) but no variation in space.

The derivation of effective parameters, given the spatial variability across the landscape, can be viewed in two ways. One is an aggregation framework in which knowledge of the local scale parameter values is used within a theoretical scaling framework to derive the landscape scale effective values. Examples of such a framework are given by
Blyth et al. (1996) and Claussen (1996). Beven (1995) has argued that such an aggregation approach will ultimately prove impossible and that it might be better to view the problem of predicting landscape scale fluxes within a disaggregation framework, in which the landscape scale model is viewed as a parameterization of the heterogeneity of fluxes within the landscape. The effective parameter approach would then be one parameterization that attempts to deal with the problem of heterogeneity in a particularly simple way based on a certain consistent linearity of fluxes. Beven (1995) points out that even a simple weighted average of a small number of models, that reflect the strong causes of heterogeneity of function (i.e. evapotranspiration flux), might be a better model when viewed from a disaggregation framework. This paper takes that approach further by exploring the responses of a relatively simple local or patch scale SVAT model in terms of the range of flux behaviours arising from the feasible ranges of the model parameter values.

Beven (1995) suggested the following form of landscape scale model:

$$
Y(t)=\sum W_{i} \Phi\left(\Theta_{i}, U, t\right) \quad i=1, N
$$

where the $Y$ values are some predicted variables, the $W_{i}$ are linear weights chosen such that $\Sigma W_{i}=1$, the $\Phi\left(\Theta_{i}, U, t\right)$ represents the predicted response of the $i$ th model with parameters $\Theta_{i}$ and input variables $U$ at time $t$ and $N$ is an 
appropriate number of models to reflect the essential aspects of variability at the landscape scale. It is worth noting that some aggregation models reflecting variability in terms of fractional areas of vegetation types or distribution functions of important parameters are essentially of this type (e.g. Avissar, 1992; Dolman, 1992; Famiglietti and Wood, 1994) but require that both model parameters and weighting coefficients be specified a priori.

In this study, the behaviour of a SVAT model when parameterised with many different feasible parameter sets is investigated. Similarity of response is observed and defined according to the cumulative evapotranspiration flux for each model simulation. Simulations are then grouped according to their functional behaviour and the posterior parameter distributions of these sets are investigated with the aim of identifying the relative sensitivity of the model parameters. This also demonstrates the potential range of parameter values that an individual parameter may be assigned and yet still produce similar behaviour dependent on the other parameter values. Structure in the posterior parameter distributions for given classes of similar model behaviour is then investigated through the application of Principal Component Analyses with the aim of investigating the nature of the covariance between the model parameters.

\section{Defining functional behaviour in a SVAT model}

The approach taken here is a disaggregation methodology in which the representation of heterogeneity of responses in the landscape is treated as a mapping of the landscape responses into an appropriate model space, where that model space is defined not so much in terms of parameter dimensions but in terms of function (i.e. flux behaviour). SVAT models have too many parameters. Previous work has shown that this leads to many different parameter sets in a SVAT model leading to similar functional behaviours, both in fitting observed data on evapotranspiration rates and in predicting cumulative evapotranspiration (and sensible heat) fluxes, a major aim of SVAT modelling (Franks et al., 1997; Franks and Beven, 1997a). Thus it is possible that many parameter sets, from those that are feasible in representing the local responses within a given landscape of interest, might predict very similar behaviours over a day, or longer periods of time. It may, therefore, not be necessary to consider all possible models in formulating the weighted sum model above, but it will be important to represent the dominant functional behaviours in the landscape and, in some cases, the extremes. In this study, functional types are defined and investigated in terms of the parameter space that defines the range of responses of the TOPUP SVAT model. This particular model is used only as an exemplar. A similar methodology can be applied to a wide range of models, from simple to complex, with the proviso that the model (or models) should be capable of simulating the range of local responses in the area of interest.

\section{A representative patch scale SVAT model: the TOPUP model}

This section describes a simple patch scale SVAT model, TOPUP (Beven and Quinn, 1994; Quinn et al., 1995). The purpose of this model is to simulate evaporative fluxes between the land surface and atmosphere. The form of this model is similar to a simple bucket type SVAT model, commonly used within GCMs, except that the lateral (downslope) redistribution of water is incorporated within the model structure. The aim has been to provide sufficient functionality in the model to reproduce the main controls on evapotranspiration, whilst minimising the number of parameters to be identified. The processes represented by the TOPUP SVAT model are illustrated in Fig. 1. The model consists of three sources from which moisture is available for evapotranspiration (see Fig. 1):

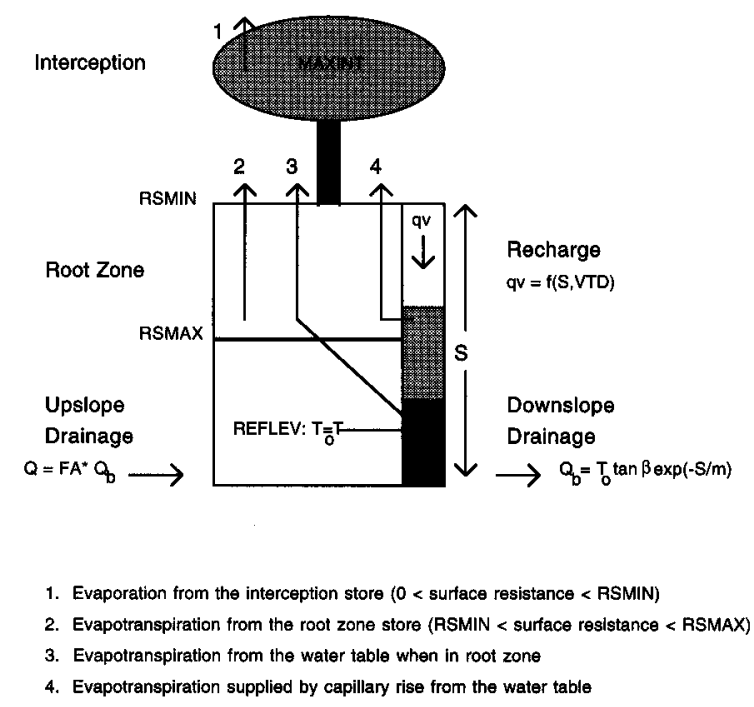

Fig. 1. Schematic representation of the TOPUP SVAT model.

a canopy/topsoil interception store (with capacity MAXINT), which, as well as representing the canopy interception store, can also serve to mimic the recovery of evapotranspiration in a dry soil following rainfall; a root zone store (with capacity SRMAX); and a variable water table. The model is based upon the Penman-Monteith equation (Monteith, 1981) requiring the specification of aerodynamic and surface resistances. Within the TOPUP SVAT model structure, surface resistance is calculated as a linear function of available moisture against storage (parameter SRMAX), between maximum and minimum resistance parameters (RSMAX and RSMIN, respectively, for evapotranspiration). Surface resistances for evaporation 
from the interception store follow a linear relationship between $0 \mathrm{sm}^{-1}$ and RSMIN according to the ratio of the available intercepted moisture and the interception store, MAXINT. Aerodynamic resistance is calculated as a function of windspeed and atmospheric instability requiring the parameterization of roughness length for momentum, $z_{0}$, zero displacement height, $d$, and the ratio of the roughness lengths for momentum and vapour, $\ln \left(z_{0} / z_{h}\right)$, (Franks et al., 1997)

The lateral subsurface flow component of the model is based on a similar set of assumptions to those used by TOPMODEL (Beven and Kirkby, 1979; Quinn and Beven, 1993; Beven et al., 1995) in which downslope fluxes are assumed to be in equilibrium with a recharge rate calculated from total hillslope discharge averaged over some area, lateral transmissivity is assumed to be an exponential function of subsurface storage and the downslope hydraulic gradient is assumed equal to the surface slope. With these assumptions, the outflow per unit contour length is given by:

$$
Q_{b}=T_{0} \tan \beta e^{-\frac{s}{m}}
$$

where $\tan \beta$ is the hydraulic gradient (assumed a constant for the given patch area), $T_{0}$ is the transmissivity of the soil when the water table is at a level given by the parameter REFLEV, $S$ is a storage deficit due to drainage of the water table below that level, and $m$ is a scaling parameter for transmissivity. Within this model structure, $T_{0}$ and $\tan \beta$ occur as a product so they may be considered as a single parameter, thereby further reducing the number of parameters to be considered. The TOPMODEL theory, where it is deemed an adequate approximation, allows the $m$ parameter to be related to the discharge recession characteristics of a catchment area. The introduction of the reference level parameter REFLEV, the depth at which $T=T_{0}$, allows the treatment of deeper water tables than with the normal version of TOPMODEL where the reference level is taken to be the soil surface (see Quinn et al., 1991). The water table is maintained from upslope by inflowing discharge calculated as a fraction of the outflow subsurface discharge according to the fractional upslope area parameter, $\mathrm{F}_{\mathrm{A}}$. Moisture routing through the unsaturated zone is achieved through a time delay per unit deficit specified by the parameter, VTD.

\section{Meteorological data}

Data sets from two sites were used in this study. Two periods for each of the sites were available. IFC-3 (August 6-21, 1987) and IFC-4 (October 5-16, 1987) from the First International Satellite Land Surface Climatology Project (ISLSCP) Field Experiment (FIFE) were employed. IFC-3 contains a storm event, whilst IFC-4 was a particularly dry period within which the tall grass vegetation canopy was reaching senescence. Meteorological forcing data and evapotranspiration measurements were also employed for an Amazonian, post-deforestation pasture site from the ABRACOS database. This site is located at Fazenda Dimona, central Amazonia. Two data sets were available from the 16 October - 2 November, 1990, and from the 29 June - 10 September, 1991. Details of the instrumentation employed at the site are given in Wright et al. (1992).

\section{Similarity of functional response}

As an example of the functional behaviour of the TOPUP SVAT model (and by implication, SVAT models in general) consider Fig. 2. This summarises the results of 10000 different TOPUP simulations with parameter sets chosen randomly from reasonable ranges (see Table 1). At this stage, each parameter has been sampled from a uniform distribution across the range, but other choices of the prior distributions, and joint distributions amongst groups of parameters, could be made. Each plot in Fig. 2 represents results across the range of a single parameter. Each dot on each plot in Fig. 2 represents one model run. Each plot therefore represents a projection of the results in the high dimension parameter space onto a single parameter axis. The results are the same in each plot and are the cumulative evapotranspiration in $\mathrm{mm}$ over a 15 day period of the 3rd Intensive Field Campaign (IFC) of the First ISLSCP Field Experiment (FIFE) in Kansas in August 1987.

It is readily seen in these figures that there are high predicted cumulative evapotranspiration fluxes across the range of most of the parameter values. There are also low cumulative evapotranspiration fluxes across the range of most of the parameter values. These differences result from the interactions of parameter values in the model. This behaviour is not unique to this site and this period. Similar plots are shown in Fig. 3 for IFC-4 of FIFE, and in Figs. 4 and 5 for the 1990 and 1991 periods of the
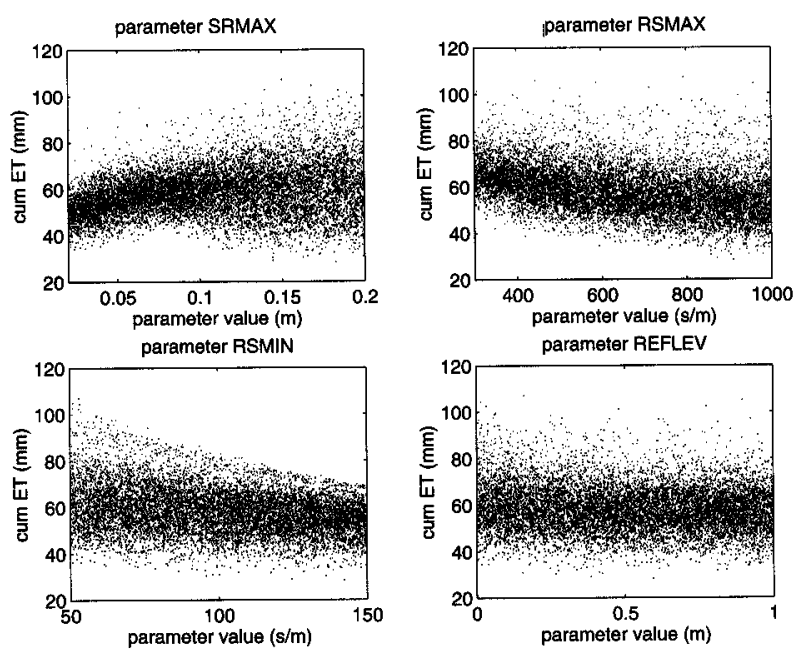

Fig. 2. Scattergrams of cumulative evapotranspiration for 4 of the model parameters when driven with FIFE IFC-3 data. 
Table 1. Parameter ranges for the TOPUP SVAT model

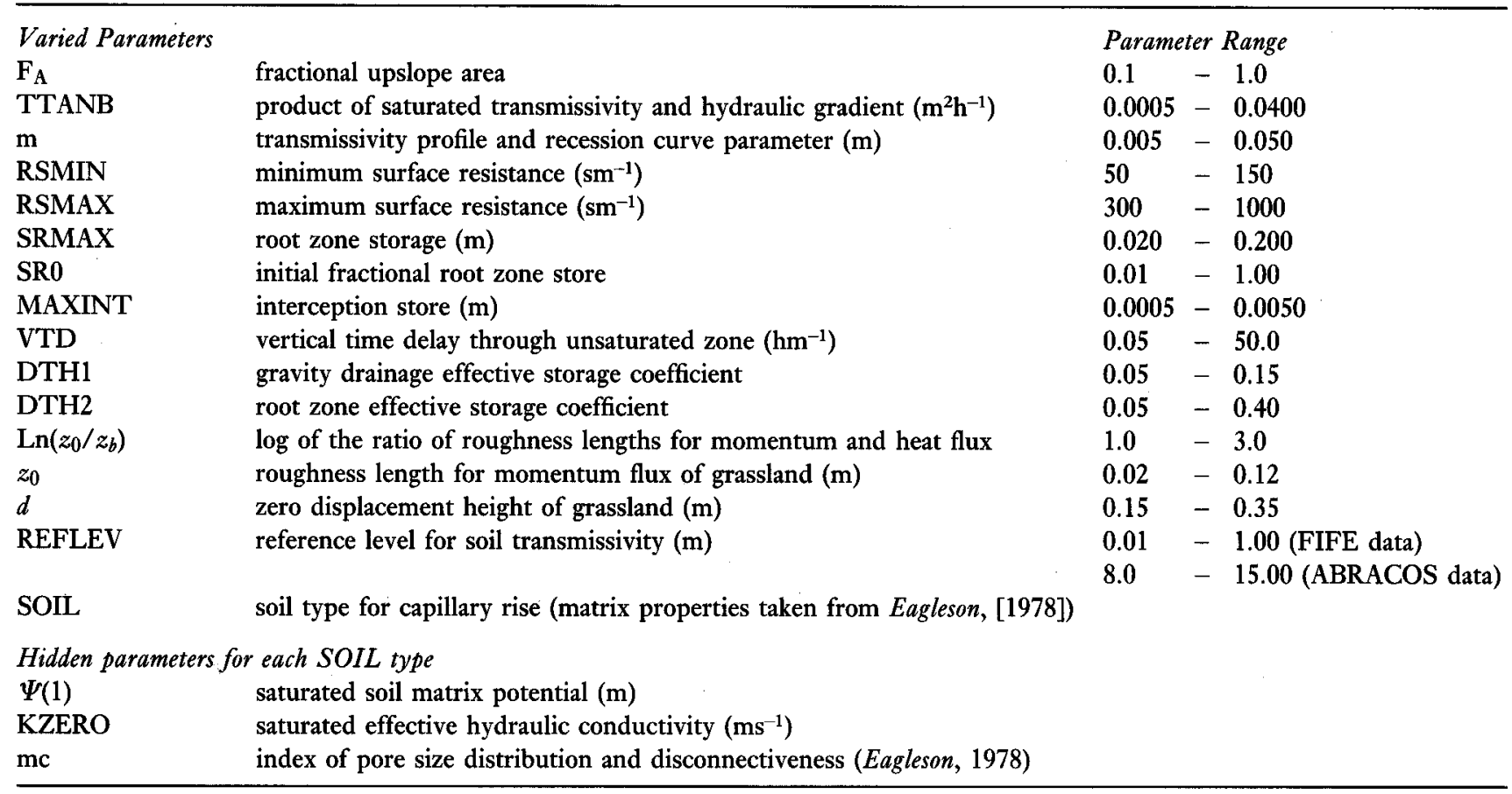

ABRACOS experiment. High fluxes and low fluxes are predicted across the parameter ranges for many parameters. In this study, the model contains a dependence of effective surface resistance on soil moisture storage but does not have any physiological feedbacks between surface resistance and atmospheric conditions which have been assumed to be the same (as measured in the field) for all model runs. If such feedbacks were included, then the variability of the ranges of cumulative evaporative flux across the individual parameter ranges would be further constrained.
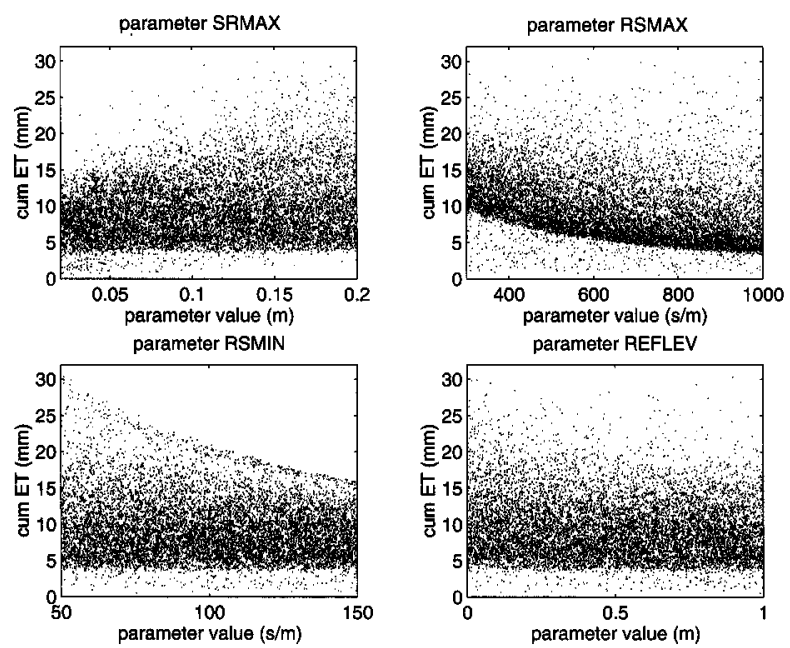

Fig. 3. Scattergrams of cumulative evapotranspiration for 4 of the. model parameters when driven with FIFE IFC-4 data.
It must be stressed that this does not imply that the model predictions are insensitive to the values of all the parameters of the model. Franks et al. (1997) have shown how this type of Monte Carlo simulation can be used to explore the sensitivity of the model to different parameter values. The parameter interactions within the non-linear model structure lead to this equifinality of behaviours in the parameter space. Such equifinality should not be unexpected given the high parameter dimensionality but it does make physical interpretation of individual parameter values rather difficult, since the effects of each parameter will
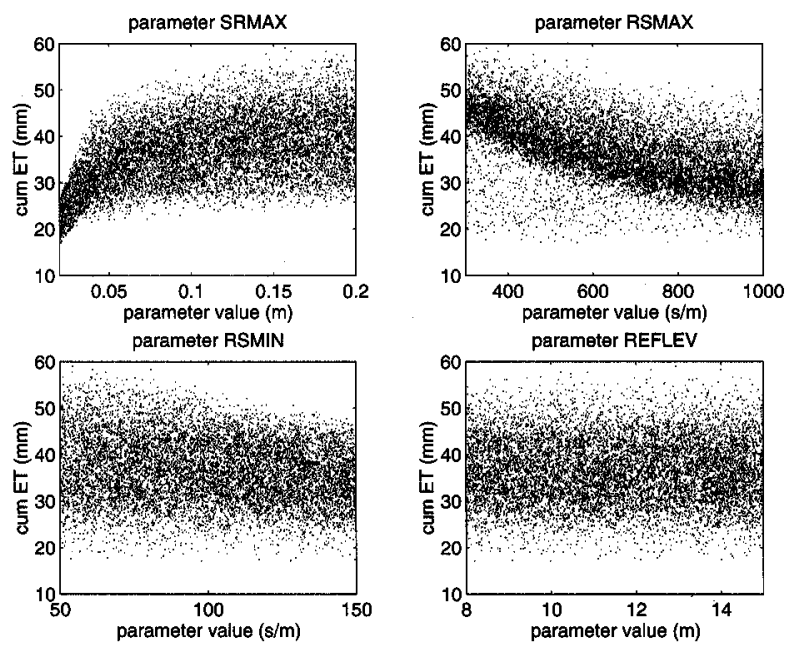

Fig. 4. Scattergrams of cumulative evapotranspiration for 4 of the model parameters when driven with ABRACOS 1990 data. 

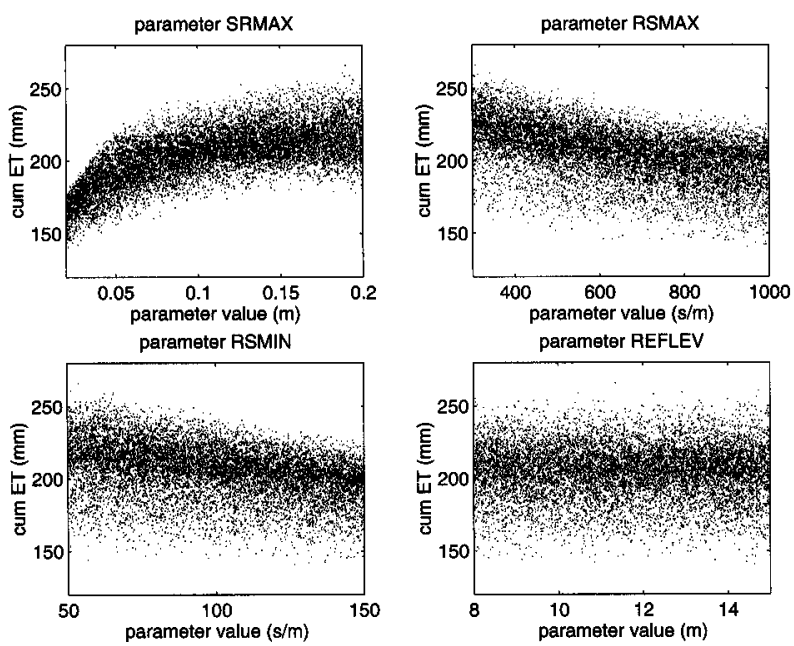

Fig. 5. Scattergrams of cumulative evapotranspiration for 4 of the model parameters when driven with ABRACOS 1991 data.

be dependent on the model structure and on the values of any other parameters.

However, the results can also be interpreted in another way. Cumulative evapotranspiration or latent heat flux can be used as one definition of the functional behaviour required of a SVAT model. It is the definition that will be used here since, in coupling the land surface to the atmosphere, a first order requirement is to predict the fluxes of energy and vapour correctly. Given the constraints of the local energy balance, then if the latent heat flux is predicted reasonably well, sensible heat fluxes should also be predicted reasonably well. Thus, it is important that some areas of the landscape are generating high latent heat fluxes, and others low latent heat fluxes, but it may not matter too much how those fluxes are predicted within the space of model behaviours as many different parameterizations result in very similar functional flux behaviour. If a given landscape could be disaggregated according to dissimilar flux behaviours, then parameterizations representative of the flux behaviours (rather than specific physical properties) might be used to simulate the flux responses of the landscape units.

Looking at the problem in this way allows the dimensionality of the parameterization to be greatly reduced, from the high dimensional space of the parameters to a low dimensional space of the model functional behaviour, however that might be defined. A definition in terms of cumulative evapotranspiration alone, as in Figs. 2 to 5, results in a single functional dimension, but other definitions can also be considered if the details of the timing of the fluxes within the period are considered important, as well as the cumulative flux.

\section{Posterior parameter distributions of functional types}

To demonstrate the wide range of parameter values that produce given functional responses for even the most sensitive of parameters, posterior distributions of each parameter for given functional types may be examined. Parameter sets were chosen by Monte Carlo sampling from uniform distributions of the parameters. As described above, the model simulations were then assigned to functional types (classes) on the basis of the cumulative evapotranspiration. Cumulative distributions of each of the parameters arising from the parameter sets that produce a cumulative evapotranspiration within a given range, defining the functional type, are then constructed. A straight line represents a uniform distribution of the parameter within that class, whilst a marked departure from the straight line would represent a non-uniform distribution. Franks et al. (1997) have used the cumulative evapotranspiration and a 'goodness of fit' measure for comparing predicted and observed fluxes as performance indicators, in a simple multivariate sensitivity analysis (see also Hornberger and Spear, 1980; Spear et al., 1994).

Posterior parameter distributions for each functional type were constructed for both FIFE and ABRACOS data sets. For each forcing data set, the model parameter sets were sub-divided into 19 groups on the basis of the cumulative evapotranspiration produced. Figure 6 shows the posterior distributions of four of the TOPUP parameters for three functional classes defined by the cumulative evapotranspiration. These three classes are classes 1,10 , and 19, representing the highest, mid and lowest functional types
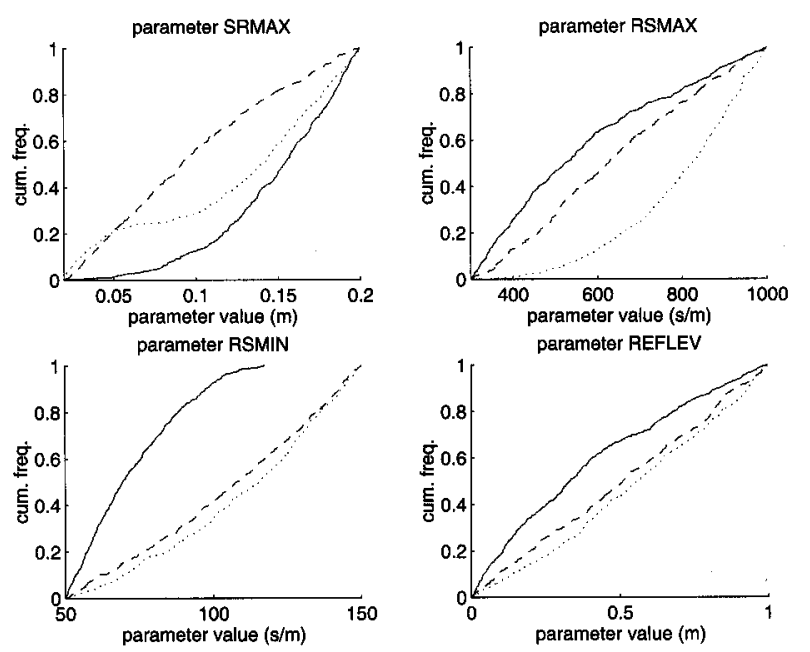

Fig. 6. Posterior distributions of four of the model parameters, for three different classes of functional behaviour as defined by the cumulative evapotranspiration when driven with the FIFE IFC-3 data set. A solid line represents class 1 (highest cum. ET class), a dashed line represents class 10 (median cum, ET class), and a dotted line represents class 19 (the lowest cum, ET class) 
with respect to the cumulative total evapotranspiration when forced with the FIFE IFC-3 period data. Figures 7, 8 and 9 show the posterior distributions of parameters for classes 1, 10 and 19, when forced with the IFC-4, ABRACOS-90 and ABRACOS-91 data sets, respectively.

Figure 6 shows the posterior parameter distributions for classes 1,10 and 19 for four of the TOPUP model parameters when forced with the IFC-3 data set. The parameter distributions for each cumulative flux class span the entire range of parameter values considered with the exception of the highest flux class for the minimum surface resistance (RSMIN) parameter. This posterior distribution is significantly non-uniform indicating relatively high sensitivity of the highest flux class to this parameter given the forcing data set.
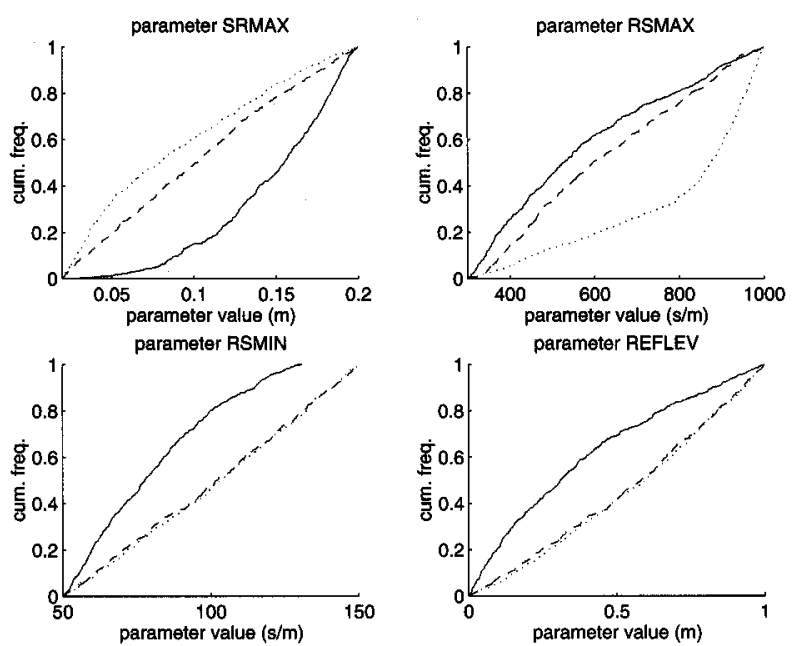

Fig. 7. Posterior distributions of four of the model parameters, for three different classes of functional behaviour as defined by the cumulative evapotranspiration mhen driven with the FIFE IFC-4 data set. A solid line represents class 1 (highest cum. ET class), a dashed line represents class 10 (median cum, ET class), and a dotted line represents class 19 (the lowest cum, ET class)

Figures 8 and 9 both show that the lowest cumulative flux class is particularly sensitive to the maximum root zone store (SRMAX) parameter, as indicated by the highly skewed posterior distributions. Indeed, the posterior distribution of the SRMAX parameter for the lowest flux class when forced with the ABRACOS-91 data set (Fig. 9) indicates that only values of this parameter less than $0.1 \mathrm{~m}$ will produce the lowest cumulative fluxes over the period of the forcing data, irrespective of the other parameter values.

The posterior distributions for the reference water table level parameter (REFLEV) for all flux classes when forced with the ABRACOS data sets (Figs. 8 and 9) can be seen to be essentially uniform. This indicates that this parameter is largely redundant in terms of influencing the cumulative totals. Reference to the posterior distributions of this
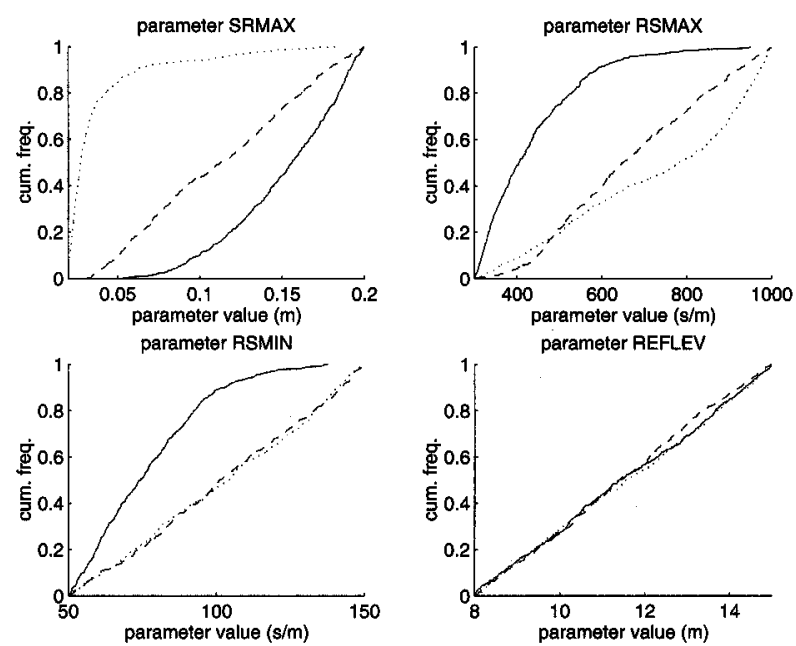

Fig. 8. Posterior distributions of four of the model parameters, for three different classes of functional behaviour as defined by the cumulative evapotranspiration when driven with the ABRACOS 1990 data set. $A$ solid line represents class 1 (highest cum. ET class), a dashed line represents class 10 (median cum, ET class), and a dotted line represents class 19 (the lomest cum, ET class)

parameter when the model is forced with the FIFE data sets, however, indicates significant sensitivity of the highest cumulative flux totals to this parameter. This difference is explained by the relatively shallow depth of the water table for the application to the FIFE site compared to the deeper level for the application of the TOPUP model to the ABRACOS site; the water table is predicted as being a significant source of moisture available for evapotranspiration at the FIFE site, whereas the deeper
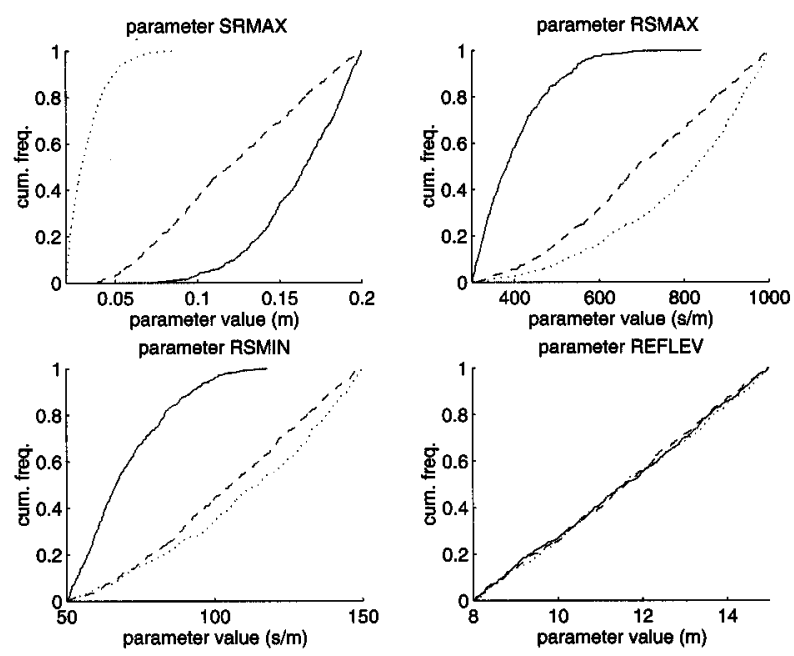

Fig. 9. Posterior distributions of four of the model parameters, for three different classes of functional behaviour as defined by the cumulative evapotranspiration when driven with the ABRACOS 1991 data set. $A$ solid line represents class 1 (highest cum. ET class), a dashed line represents class 10 (median cum, ET class), and a dotted line represents class 19 (the lowest cum, ET class) 
water table encountered at the ABRACOS site contributes significantly less moisture for evapotranspiration.

As can be seen by reference to each of the posterior distributions of the REFLEV parameter when the TOPUP model is forced with each of the meteorological data sets, values from each of the a priori ranges may produce similar cumulative flux responses due to the interactions and values of the other model parameters. This can be seen in the majority of cases, as most posterior distributions cover the entire parameter ranges indicating strong parameter interactions.

\section{Identifying parameter covariation: Principal Component Analysis}

An alternative approach to investigate the posterior distributions of parameters for each of the defined functional types is to perform a Principal Component Analysis (PCA) on the posterior distributions. The PCA approach identifies eigenvectors that explain a proportion of the variance of the data set in ranked order. These eigenvectors represent correlations in the variance of the parameter values. The importance of each such eigenvector and, hence, the importance of the corresponding parameter covariation, may be evaluated in terms of the proportion of variance explained. PCA analysis was performed on classes 1,10 , and 19 , to identify eigenvectors from the posterior parameter space. Figure 10 shows the proportion of variance explained by each of the eigenvectors for class 1 (highest cumulative evapotranspiration fluxes) when forced with the FIFE and ABRACOS data sets. In all cases only the first eigenvector has any discernible importance in terms of the proportion of the variance explained, with respect to the other eigenvectors. Even the first component explains only a small proportion of the total variance sug-
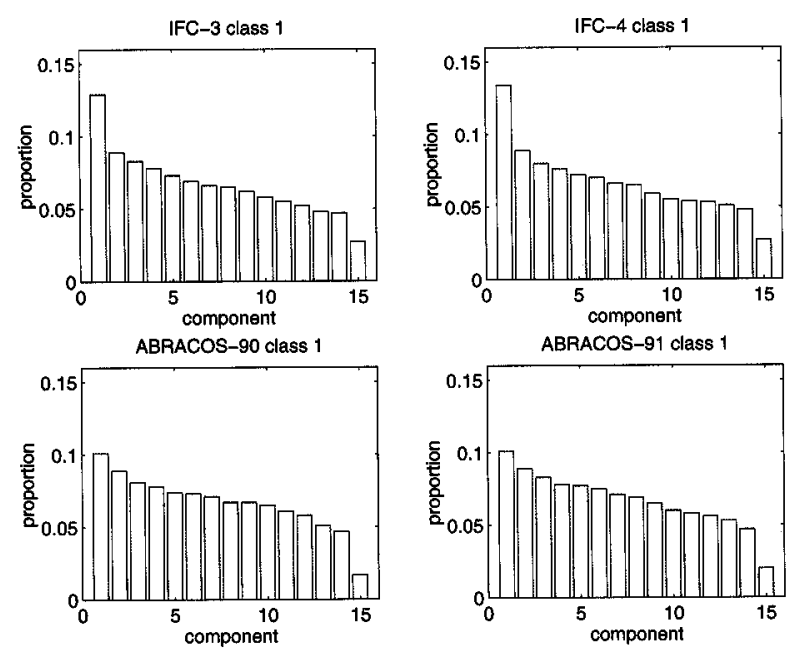

Fig. 10. Bar charts of the proportion of variance explained by each of the identified eigenvectors for the class 1 functional types when forced with each data set. gesting that there is no strong linear structural covariation in the parameter space in this class.

The PCA analysis was also performed for classes 10 and 19 resulting in remarkably uniform eigenvectors in every case. The percentages of variance explained by the first three (ranked) components for these flux classes are shown in Table 2. The lack of a dominant eigenvector is a direct result of the complex and nonlinear parameter interactions that lead to the different functional responses. There is little dependence on given eigenvectors because of the lack of strong global correlation between individual parameters of the model (though functionally there must be local interactions as the effect of a change in one parameter is compensated by changes in the other parameters).

Depending on the value of other parameters in a set, a wide range of values of any single parameter may produce similar responses as defined by the cumulative evapotranspiration for that period.

Table 2. Percentage of variance explained by the first three components for highest, median and lowest cumulative flux classes (classes 1, 10 and 19, respectively), when forced with the FIFE IFC-3 and IFC-4, and ABRACOS '90 and '91 data sets.

\begin{tabular}{llccc}
\hline Class & Forcing data set & \multicolumn{3}{c}{ Percentage of Variance Explained (\%) } \\
Components \\
& & 1st & 2nd & 3rd \\
\hline 1 & & 12.9 & 8.9 & 8.3 \\
1 & IFC-3 & 13.4 & 8.9 & 8.0 \\
& IFC-4 & 10.1 & 8.9 & 8.1 \\
& ABRACOS '90 & 10.1 & 8.9 & 8.3 \\
& ABRACOS '91 & 10.1 & 8.7 & 8.2 \\
10 & IFC-3 & 10.9 & 8.5 & 8.0 \\
& IFC-4 & 12.5 & 8.7 & 8.0 \\
& ABRACOS '90 & 11.7 & 8.5 & 8.0 \\
& ABRACOS '91 & 10.8 & 9.5 & 8.2 \\
19 & IFC-3 & 10.5 & 8.4 & 8.2 \\
& IFC-4 & 10.3 & 8.5 & 7.5 \\
& ABRACOS '90 & 12.8 & 8.6 & 8.3 \\
\hline
\end{tabular}

\section{Discussion}

For a given landscape, many of the parameter combinations selected by the Monte Carlo sampling strategy may be absent. This study has investigated the possible, rather than the probable, variations of parameter values. To what degree possible combinations do exist in a landscape is a problem that cannot be resolved easily as many parameters used in SVAT models are highly variable in space and may not easily be measured. This is a key point; aggregation strategies will be subject to high uncertainty due to the difficulties in measuring land surface characteristics. This 
study has, therefore, concentrated on the parameterization and function of SVAT models rather than on the application to a given domain.

The implications of this study on the parameterization of SVAT models are apparent: when achieved through calibration (model inversion) parameter values reported in the scientific literature will be dependent upon the other values of the other parameters. Reasonable values from various sources may produce the right (or wrong) results. Model parameterization should consider the parameter set, rather than any specific parameter, as it is the set that defines the model behaviour. It could be argued that calibration of such models might be better achieved through the use of longer time series incorporating more of the dynamics of the natural system. Whilst this is true, there remains a certain intractability due to the over-parameterization of these models: the measured fluxes (as well as containing high degrees of noise) do not contain sufficient information for the inversion of a complex model to yield robustly identified, physically-meaningful parameter values.

In terms of the results presented in this study, if longer forcing data sets were employed, then one might require the definition of functional type to incorporate the temporal variations of evapotranspiration. One could perhaps define functional types based upon a similarity of temporal responses to the meteorological forcing data. Parameter interactions might then be investigated in a similar manner to those presented here. However, this may also introduce the requirement for additional parameterization of the physiological changes of the vegetation over such long periods. Under such circumstances, it would be expected that the possibility of simulating an observed flux time series would increase as the dimensionality of the parameter space increased. It is apparent that the dimensionality of a model parameter space should be minimised to the degree that is supported by the available data: the model dimensionality should permit sufficient functionality to simulate the range of expected (preferably measured) responses in time and space.

\section{A new approach}

This study of functional types reflects the need to represent the heterogeneity of land surface responses. Complex, high-dimensional models can produce very similar responses in terms of the required function of the model with parameterizations from many different areas of the parameter space. As the aggregation of parameter fields is subject to high uncertainty due to high parameter uncertainty and the highly complex internal behaviour of physically based SVAT models, this study advocates a different approach to the representation of complex terrain. This approach is the mapping of the functional behaviour into low dimensional functional space. In principle, any overparameterised high-dimensional complex model may be investigated in terms of its function. The functional space may then be constrained for any given application through whatever information is available. In terms of this study, the aim is to parameterise variable surface fluxes at the landscape scale. Given a range of possible functionally different behaviours arising from the feasible parameter space, one can identify areal weightings for significantly different functional types through the use of distributed thermal remotely-sensed data. This is explored by Franks and Beven (1997b), within an information-based uncertainty framework, whereby uncertain estimates of distributed fluxes are used to condition a multiple patch SVAT model (as represented by Eqn. 1).

\section{Acknowledgements}

This work was achieved through the financial support provided by the NERC TIGER (Terrestrial Initiative in Global Environment Research) programme. Thanks are due to Ivan Wright for supplying the ABRACOS data sets, and to all those people involved in the collection and compilation of both the FIFE and ABRACOS data sets.

\section{References}

Avissar, R., (1992). Conceptual aspects of a statistical-dynamical approach to represent landscape scale sub-grid heterogeneities in atmospheric models, 7. Geophys. Res., 97(D3), 2729-2742.

Beven, K.J., (1995). Linking parameters across scales: sub-grid parameterizations and scale dependent hydrological models, Hydrol. Process., 9, 507-526.

Beven, K.J. and Kirkby, M.J., (1979). A physically based variable contributing area model of basin hydrology, Hydrol. Sci. Bull., 24, 43-69.

Beven, K.J., and Quinn, P.F., (1994). Similarity and scale effects in the water balance of heterogeneous areas, Proc. AGMET conference on The Balance of Water-Present and Future, AGMET, Dublin. September 1994.

Beven, K.J., Lamb, R., Quinn, P.F., Romanowicz, R. and Freer, J., (1995). TOPMODEL, in Singh, V.P., (Ed.), Computer models of watershed hydrology, Water Resource Publications, Highlands Ranch, Co.

Blyth, E.M., Dolman, A.J. and Wood, N., (1993). Effective resistance of sensible and latent heat flux in heterogeneous terrain, Quart. 7. Roy. Meteorol. Soc., 119, 423-442.

Claussen, M., (1995). Flux aggregation at large scales: on the limits of validity of the concept of blending height, 7. Hydrol., 166, 371-382.

Dickinson, R.E. and Kennedy, P.J., (1991). Land surface hydrology in a general circulation model-global and regional fields needed for validation, in Wood, E.F. (Ed.), Land SurfaceAtmosphere Interactions for Climate Modelling, Kluwer, Dordrecht, 115-126.

Dolman, A.J., (1992). A note on areally-averaged evaporation and the value of effective surface conductance, 7 . Hydrol., 138, 583-589.

Eagleson P.S., (1978). Climate, soil and vegetation. 3. A simplified model of soil moisture movement in the liquid phase, Wat. Resour. Res., 14, 722-730. 
Entekhabi, D. and Eagleson, P.S., (1989). Land surface hydrology parameterization for atmospheric general circulation models including subgrid scale spatial variability, f. Climate, 2(8), 816-831.

Famiglietti, J.S. and Wood, E.F., (1994). Multiscale modelling of spatially variable water and energy balance processes, Wat. Resour. Res., 30, 3061-3078.

Franks, S.W. and Beven, K.J., (1997a). Bayesian estimation of uncertainty in land surface-atmosphere flux predictions, 7 . Geophys. Res., 102 (D20), 23991-23999.

Franks, S.W. and Beven, K.J., (1997b). Modelling evapotranspiration at the landscape scale: a fuzzy disaggregation approach, Wat. Resour. Res., 33, 2929-2938.

Franks, S.W., Beven, K.J., Quinn, P.F. and Wright, I.R., (1997). On the sensitivity of Soil Vegetation-Atmosphere Transfer (SVAT) schemes: equifinality and the problem of robust calibration, Agric. For. Meteorol., 86, 63-75.

Monteith, J.L., (1981). Evaporation and surface temperature. Quart. 7. Roy. Meteorol. Soc., 107, 1-27.

Quinn, P.F., Beven, K.J., Chevallier, P. and Planchon, O., (1991). The prediction of hillslope flow paths for distributed hydrological modelling using digital terrain models. Hydrol. Process., 5, 59-79.
Quinn, P.F. and Beven, K.J., (1993). Spatial and temporal predictions of soil moisture dynamics, runoff, variable source areas and evapotranspiration for Plynlimon, mid- Wales, Hydrol. Process., 7, 425-448.

Quinn, P.F., Beven, K.J. and Culf, A., (1995). The introduction of macroscale hydrological complexity into land surfaceatmosphere transfer models and the effect on planetary boundary layer development. 7. Hydrol., 166, 421-444.

Sellers, P.J., Mintz, Y., Sud, Y.C. and Dalcher, A., (1986). A simplified biosphere model ( $\mathrm{SiB}$ ) for use within general circulation models, 7. Atmos. Sci., 43, 305-331.

Spear, R.C. and Hornberger, G.M., (1980). Eutrophication in Peel Inlet II. Identification of critical uncertainties via Generalised Sensitivity Analysis, Water Res., 14, 43-49.

Spear, R.C., Grieb, T.M. and Shang, N., (1994). Parameter uncertainty and interaction in complex environmental models, Wat. Resour. Res., 30, 3159-3169.

Wright I.R., Gash, J.H.C., Da Rocha, H.R., Shuttleworth, W.J., Nobre, C.A., Maitelli, G.T., Zamparoni, C.A.G.P. and Carvalho, P.R.A., (1992). Dry season micrometeorology of central Amazonian ranchland. Quart. 7. Roy. Meteorol. Soc., 118: 1083-1099. 